\title{
Embrittlement of Mn-Si and Mn-Mo High Strength Steels Caused by Heating in High Pressure Hydrogen at High Temperatures*
}

\author{
By Tadashi Nemoto**, Kazuo Tanosaki** and Isao Masaoka**
}

\begin{abstract}
Three kinds of high strength steel were heated for differing periods of time in $200 \mathrm{~kg} / \mathrm{cm}^{2}$ hydrogen at high temperatures of $300 \sim 500^{\circ} \mathrm{C}$, and the changes in their mechanical properties and structures were studied at room temperature. The results obtained are as follows: (1) The steels which were heated in high pressure hydrogen at high temperatures became brittle and showed a remarkable decrease in the mechanical strength and ductility. The concurrent decrease in both hardness and carbon content occurred with many inner cracks at grain boundaries. The fissures at grain boundaries gave rise to deterioration in the quality of materials. (2) At a constant temperature and pressure, there was an incubation time $(t)$ prior to embrittlement and a plot of the $\ln t$ vs. reciprocal of absolute temperature holds a linear relationship. (3) The activation energy of embrittlement on the kinetics of hydrogen attack was calculated, but the mechanism of embrittlement by hydrogen attack could not be clearly explained. (4) The addition of molybdenum, a carbide forming element and such heat treatments as normalizing and tempering increase the resistance to hydrogen attack and raise embrittlement limits.
\end{abstract}

(Received October 20, 1964)

\section{Introduction}

Brittle failures of steels caused by high pressure hydrogen gas at elevated temperatures have been encountered in chemical industry dealing with ammonia synthesis, methanol synthesis, liquefaction of coal, petroleum manufacturing, etc.

This embrittlement is called "hydrogen attack", and this phenomenon was already found about thirty years ago by Naumann(1)(2), Inglis(3) and Okura(4). But these investigators mainly dealt with the effect of alloying elements upon the embrittlement of forging materials after heating for about $100 \mathrm{hr}$ in high pressure hydrogen at high temperatures. The reports on the process of embrittlement by a long-time observation or on the embrittlement limits of steels are few, and Nelson ${ }^{(5)(6)}$ obtained only safe operating limits of carbon and alloy steels from the actual results.

In the present ievestigaion, $\mathrm{Mn}-\mathrm{Si}$ and $\mathrm{Mn}-\mathrm{Mo}$ high strength steels were heated in high pressure hydrogen at high temperatures, and the embrittlement process was studied by measuring the changes in their mechanical properties and structures.

\section{Specimens and Experimental Procedure}

The specimens used were three commercial high strength steels ; Mn-Si type Steels A and B and Mn-Mo type Steel C. The chemical compositions of the specimens are shown in Table 1 and their microstructures in Photo. 1. Steels A and C are as hot rolled (thickness :

Table 1 Chemical composition of specimens (\%).

\begin{tabular}{c|c|c|c|c|c|c|c|c|c|c}
\hline \hline Strel & $\mathrm{C}$ & $\mathrm{Si}$ & $\mathrm{Mn}$ & $\mathrm{P}$ & $\mathrm{S}$ & $\mathrm{Cu}$ & $\mathrm{Ni}$ & $\mathrm{Cr}$ & $\mathrm{Mo}$ & $\mathrm{V}$ \\
\hline $\mathrm{A}$ & 0.19 & 0.47 & 1.40 & 0.023 & 0.009 & 0.20 & $\mathrm{tr}$ & 0.13 & 0.04 & 0.07 \\
$\mathrm{~B}$ & 0.16 & 0.19 & 1.15 & 0.021 & 0.007 & 0.16 & $\mathrm{tr}$ & 0.07 & 0.03 & 0.05 \\
$\mathrm{C}$ & 0.14 & 0.26 & 1.11 & 0.016 & 0.004 & 0.14 & $\mathrm{tr}$ & 0.16 & 0.58 & 0.05 \\
\hline
\end{tabular}

* This paper was pablished in Japanese in the Journal of the Japan Institute of Metals, 27 (1963), 177.

** Hitachi Research Laboratory, Hitachi Ltd., Ibaragi Prefecture, Japan.

(1) F. K. Naumann : Stahl u. Eisen, 57 (1937), 889
(2) F. K. Naumann : Stahl u. Eisen, 58(1938), 1239.

(3) N. P. Inglis : J. Iron Steel Inst., 128(1933), 383.

(4) S. Okura : J. Iron Steel Inst. Japan, 28 (1942), 30

(5) G. A. Nelson : Trans. ASME, 73 (1951), 205.

(6) G. A. Nelson : Trans. ASME, 81 (1959), 92. 
$25 \mathrm{~mm}$ ) and their microstructures consist of a mixture of ferrite and pearlite, but Steel B is normalized and tempered after rolling (thickness : $46 \mathrm{~mm}$ ). The mechanical properties were examined on tensile test pieces 50 $\mathrm{mm}$ in gauge length, $10 \mathrm{~mm}$ in width and $5 \mathrm{~mm}$ in shown in Fig. 2, with a tendency similar to that in Fig. 1. Above the critical heating temperature, the steels suddenly lose their ductility and decrease their impact values. But the critical temperature for embrittlement in the impact properties is lower than that for the tensile

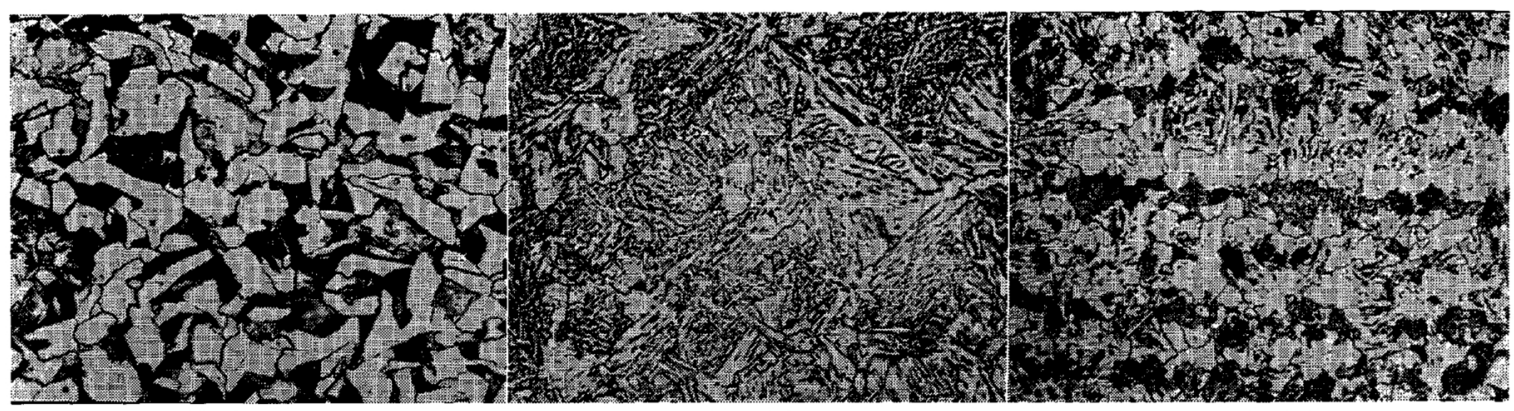

Steel A
Steel B

Steel C

Photo. 1 Structure of specimens; Etching, 5\% Picral . ( $\times 160)$

thickness and on standard Charpy test bars with a $2 \mathrm{~mm}$ $\mathrm{V}$-notch. The specimens were machined from the abovementioned thick plates and annealed in vacuum at $650^{\circ} \mathrm{C}$ for $1 \mathrm{hr}$.

The specimens were inserted in $2 \mathrm{~L}$ autoclaves and heated after charging of high pressure hydrogen. The capacity of the autoclaves was : maximum pressure, 400 $\mathrm{kg} / \mathrm{cm}^{2}$ and maximum temperature, $600^{\circ} \mathrm{C}$. The pressures were measured by means of Bourdon tube pressure gauges, and the temperatures were automatically recoreded by thermocouples with protective tubes leading to the center of the autoclaves.

The effects of heating temperature and time upon the mechanical properties and microstructures were examined at room temperature after heating for various times up to $1000 \mathrm{hr}$ in $200 \mathrm{~kg} / \mathrm{cm}^{2}$ hydrogen at temperatures of $300 \sim 550^{\circ} \mathrm{C}$. Commercial bomb hydrogen gas (dew point $-20^{\circ} \mathrm{C}$, was introduced into the autoclaves without drying.

\section{Experimental Results and Discussion}

\section{The changes in mechanical properties by heating in high pressure hydrogen at high temperatures}

Fig. 1 shows the effect of heating temperatures on the mechanical properties of Steels A, B and C after heating in $200 \mathrm{~kg} / \mathrm{cm}^{2}$ hydrogen for $100 \mathrm{hr}$. Under this condition, Steel A shows no change in mechanical properties up to the heating temperature of $350^{\circ} \mathrm{C}$, but above $400^{\circ} \mathrm{C}$ the tensile strength and ductility decrease remarkably and the elongation and reduction of area become zero. Above $400^{\circ} \mathrm{C}$ the tensile strength is settled in the range of $30 \sim 35 \mathrm{~kg} / \mathrm{mm}^{2}$, which is nearly equal to the strength of extra soft steels. In Steel B the decrease of strength occurs on the higher temperature side, and the steel is known to have higher resistance to the embrittlement than Steel A. Steel B becomes brittle above $450^{\circ} \mathrm{C}$. Steel $\mathrm{C}$ has no tendency of embrittlement up to $500^{\circ} \mathrm{C}$ under this condition, and a much severer condition seems necessary to produce the embrittlement in tensile properties.

The changes in impact values of these three steels are

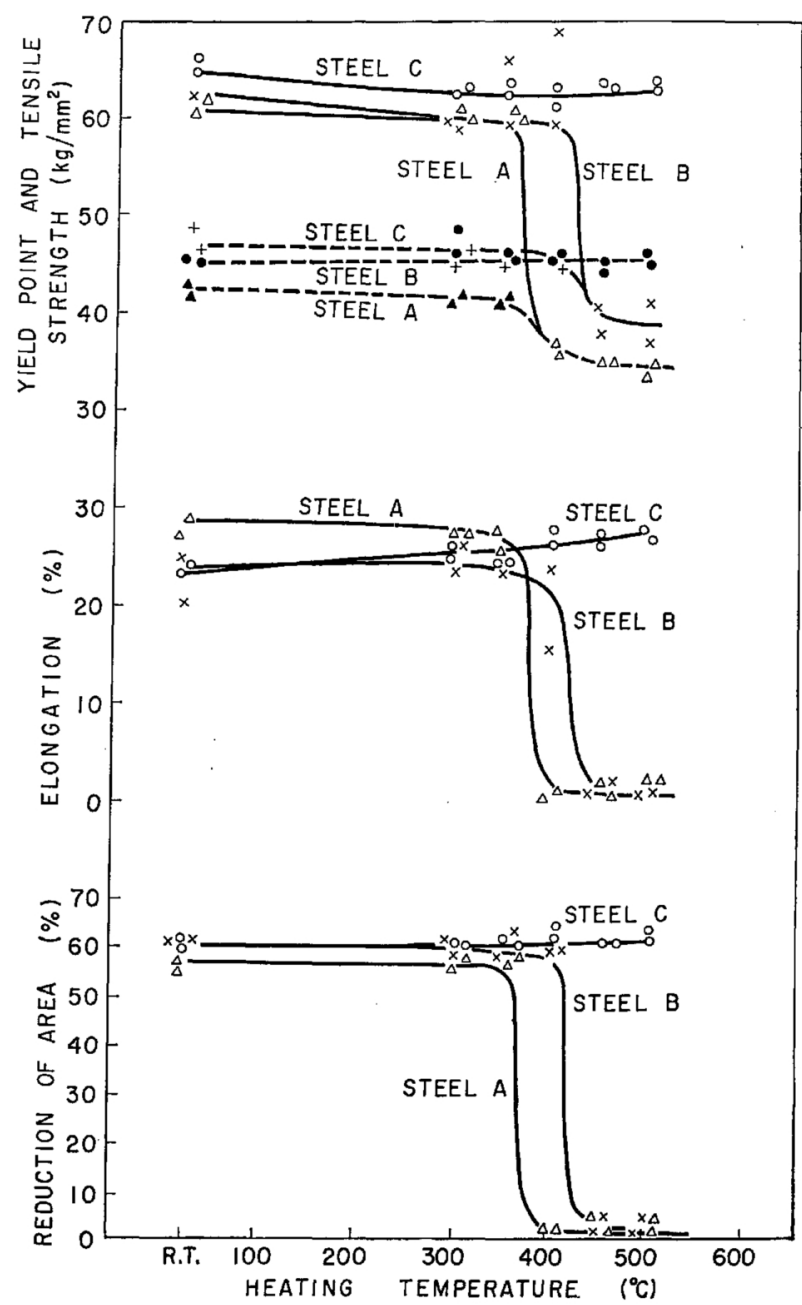

Fig. 1 Effect of heating temperature on mechanical properties after heating for $100 \mathrm{hr}$ in $200 \mathrm{~kg} / \mathrm{cm}^{2}$ hydrogen.

properties shown in Fig. 1. This seems to be caused by the higher structure sensivity of the impact properties than the tensile properties. The results by heating for $1000 \mathrm{hr}$ (Fig. 2) show that the embrittlement temperature is $300^{\circ} \mathrm{C}$ for Steel A and $400^{\circ} \mathrm{C}$ for Steel B, but Steel C holds a relatively high impact value at 400 ${ }^{\circ} \mathrm{C}$ at which there is a sign of embrittlement.

An observation of the fracture surface of the specimens, shows that the ductile fracture surfaces have a 
typical fibrous appearance, but the brittle fracture surfaces have no bright cleavages often observed in low temperature brittle fractures but a characteristic dull grey intercrystalline fracture appearance.

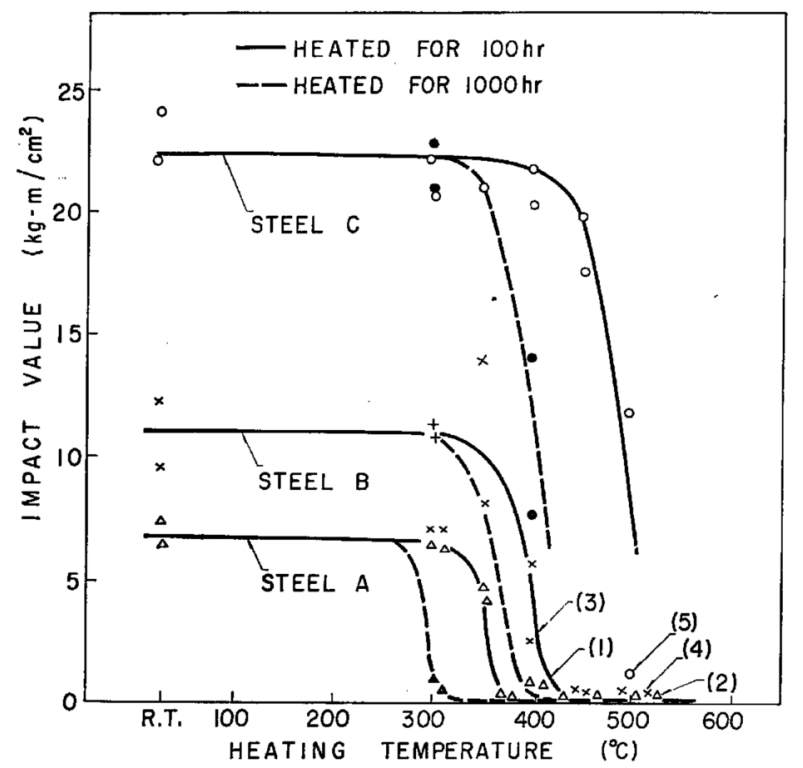

Fig. 2 Effect of the heating temperature on the impact value after heating for $100 \mathrm{hr}$ and $1000 \mathrm{hr}$ in $200 \mathrm{~kg} / \mathrm{cm}^{2}$ hydrogen.

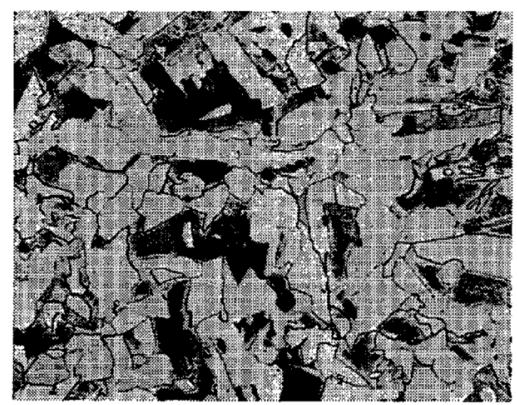

(a) Specimen (1) in Fig. 2

Fhoto. 2

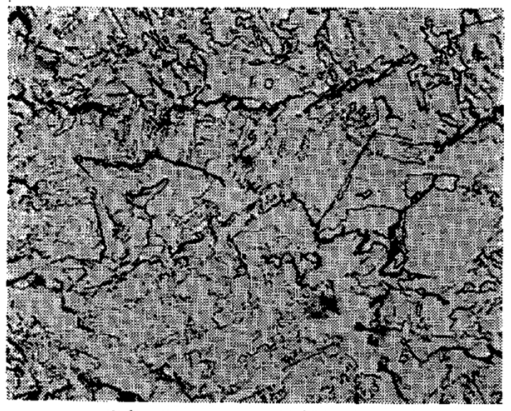

(a) Specimen (3) in Fig. 2

\section{The changes of microstructures after heating in high pressure hydrogen at high temperatures}

Photos. 2 4 shows the microstructures of specimens after impact tests corresponding to specimen $(1) \sim(5)$ o fFig. 2

Photo. 2 shows microstructures of Steel A. In the specimen heated at $400^{\circ} \mathrm{C}$ (Fig. $2(1)$ ), pearlites decompose in the vicinity of grain boundaries, and inner cracks initiate at grain boundaries. At $500^{\circ} \mathrm{C}$ (in Fig. 2(2)), pearlites decompose completely and are decarburized, and complete separations and cracks at grain boundaries are visible. This is obvious from the pattern in which the etching reagent is diffused.

Photo. 3 shows microstructures of Steel B. At $400^{\circ} \mathrm{C}$, at which the impact value began to decrease, fine cracks along grain boundaries and leaves of bainites are found. But in the specimens embrittled completely at $500^{\circ} \mathrm{C}$, bainite decomposition and many inner cracks are clearly observed.

Photo. 4 shows microstructures of Steel C. This steel has relatively high resistance to the initiation of inner cracks, and the inner cracks are seen in some specimens which are embrittled at $500^{\circ} \mathrm{C}$. No structural change is observed even in the specimens with partially reduced

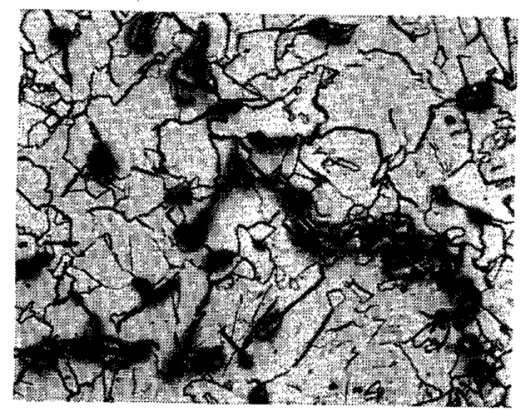

(b) Specimen (2) in Fig. 2

Steel A

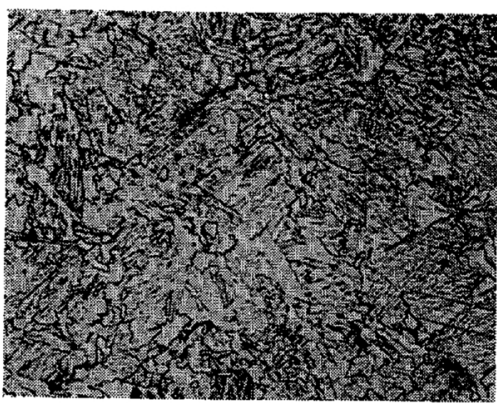

(b) Specimen (4) in Fig. 2

Photo. 3 Steel B

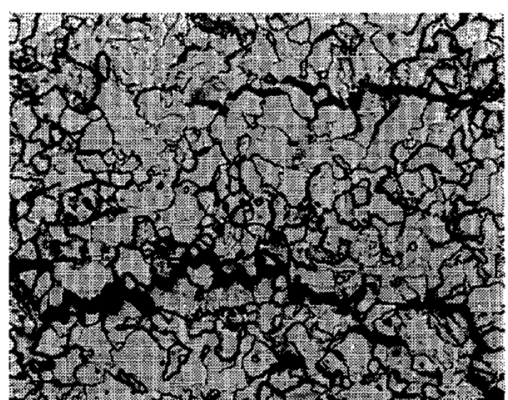

Specimen (5) in Fig. 2

Photo. 4 Steel C

Photo. 2 4 Structure change after heating in $200 \mathrm{~kg} / \mathrm{cm}^{2}$ hydrogen, Etching, $5 \%$ Picral. $(\times 160)$ 
impact values, indicating that impact properties are sensitive to the initiation of the smallest cracks.

\section{The changes in the hardness, carbon content and weight loss}

The results of examination on the Vickers hardness, carbon content and weight loss of the impact tested specimens in Fig. 2 are shown in Fig. 3. The Vickers

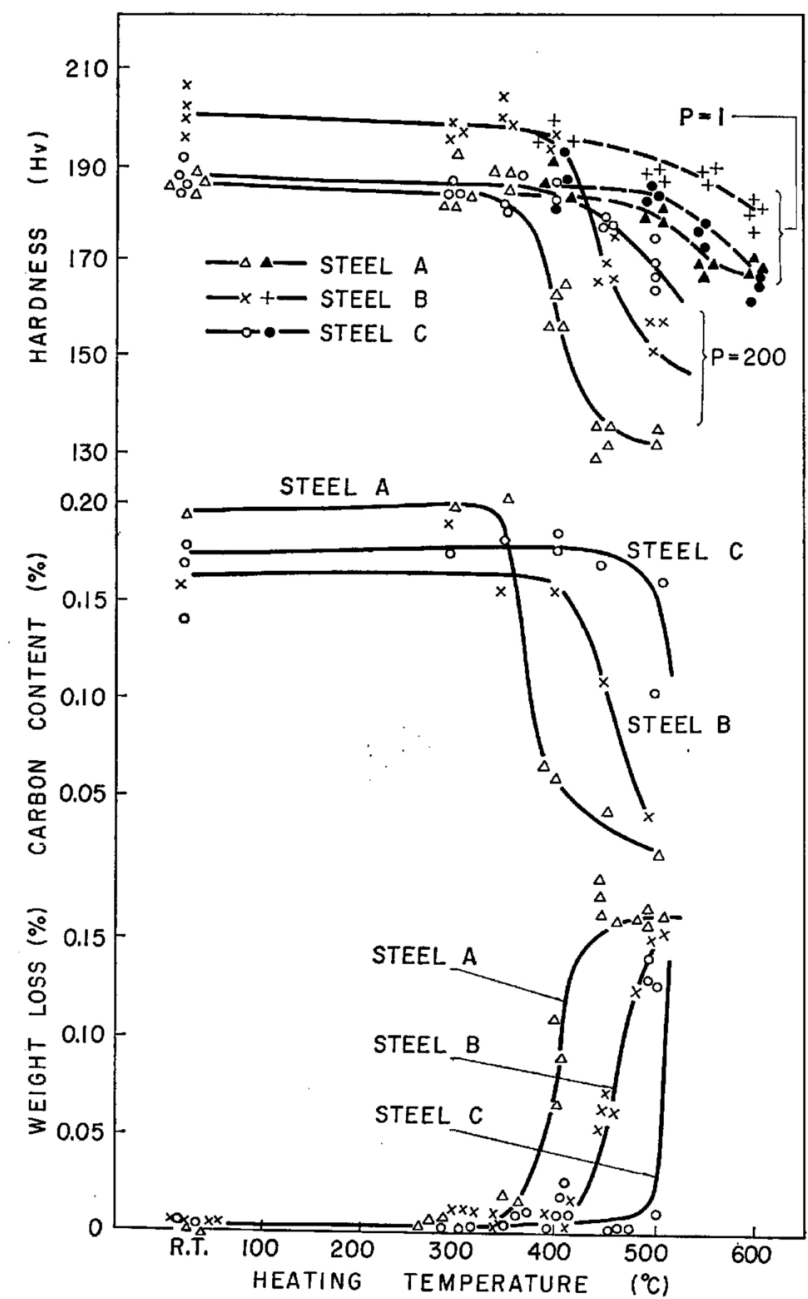

Fig. 3 Effect of heating temperature on hardness, weight change and carbon content after heating for $100 \mathrm{hr}$ in $200 \mathrm{~kg} / \mathrm{cm}^{2}$ hydrogen.

hardness decreases remarkably with the reduction in impact value, which is more remarkable than the softening at the pressure of $1 \mathrm{~kg} / \mathrm{cm}^{2}$. At the same time the decreases in the carbon content and the weight loss by decarburization are also observed.

\section{The time effect on mechanical properties after heating in high pressure hydrogen at high temperatures}

When the steels are heated in high pressure hydrogen at a critical temperature for embrittlement limit temperature exists under this condition, and when they are held at a constant temperature, it was found that embrittlement begins rapidly after a definite heating time. Fig.4 shows the embrittlement process of Steel A by the changes in mechanical properties at room temperature after heating in $200 \mathrm{~kg} / \mathrm{cm}^{2}$ hydrogen for various periods of time at temperatures of $300 \sim 500^{\circ} \mathrm{C}$. At $300^{\circ} \mathrm{C}$ no decreases in tensile strength, elongation and reduction of area are observed after holding for $100 \mathrm{hr}$, but after holding for $1000 \mathrm{hr}$ a partial embrittlement begins to occur. However, at $400^{\circ} \mathrm{C}$ the ductility begins to decrease by heating for about $10 \mathrm{hr}$, and a complete loss in ductility is observed by heating for $100 \mathrm{hr}$. Moreover, at $450^{\circ} \mathrm{C}$ embrittlement begins after a short time heating, and it is obvious that as the heating temperature rises,

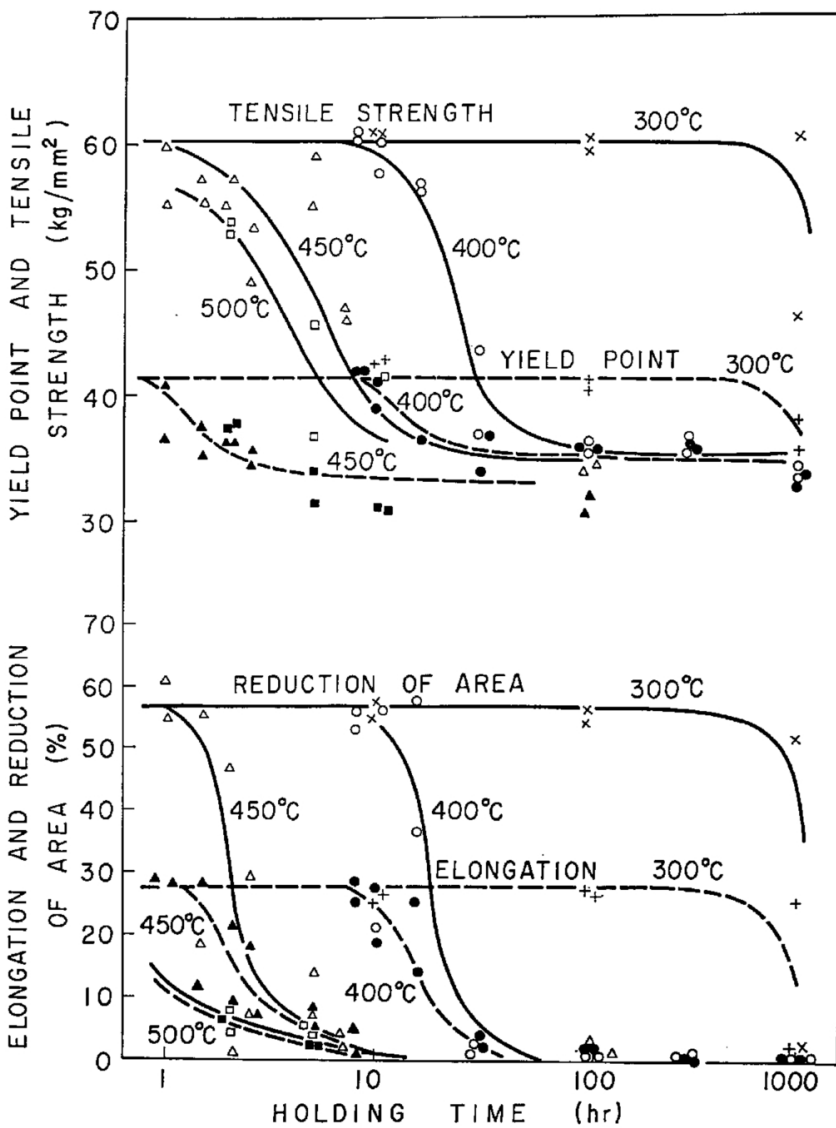

Fig. 4 Change in mechanical properties of Steel A after heating in $200 \mathrm{~kg} / \mathrm{cm}^{2}$ hydrogen.

the embrittlement beginning time becomes shorter. The tensile strength values scatter considerably at high temperatures. On the other hand, the ductility decreases remarkably in a short time when the embrittlement

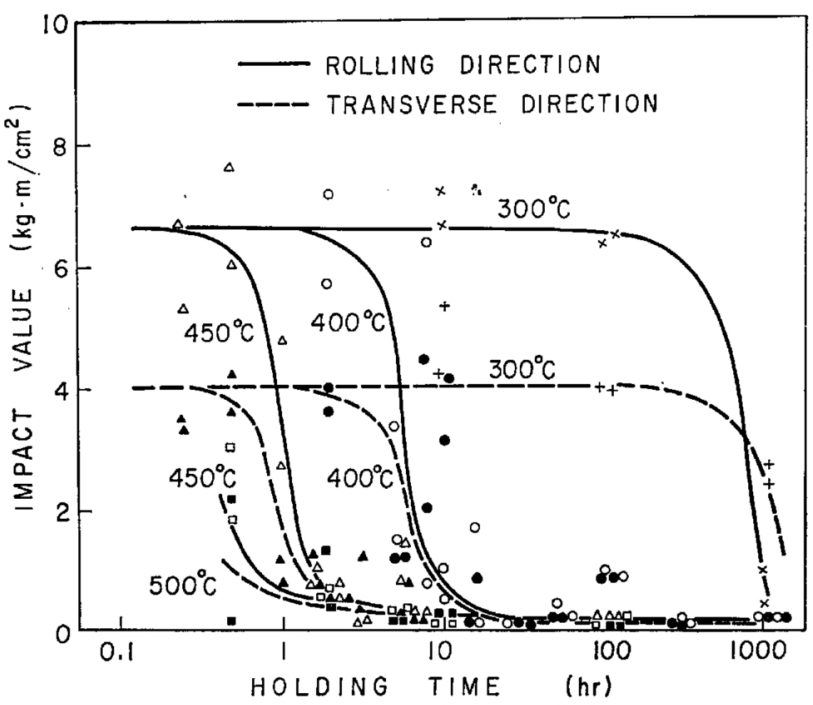

Fig. 5 Change in impact value of Steel $A$ after heating in $200 \mathrm{~kg} / \mathrm{cm}^{2}$ hydrogen. 
begins.

As can also be seen from the impact test result in Fig. 5 , the time required for brittlement at low temperatures is considerably longer than that at high temperatures. It is noted that the time required for embrittlement in has the lowest embrittlement limit and Steel C has the highest resistance to hydrogen attack. The embrittlement limits obtained from the elongation and reduction of area are somewhat lower than that from the impact value.

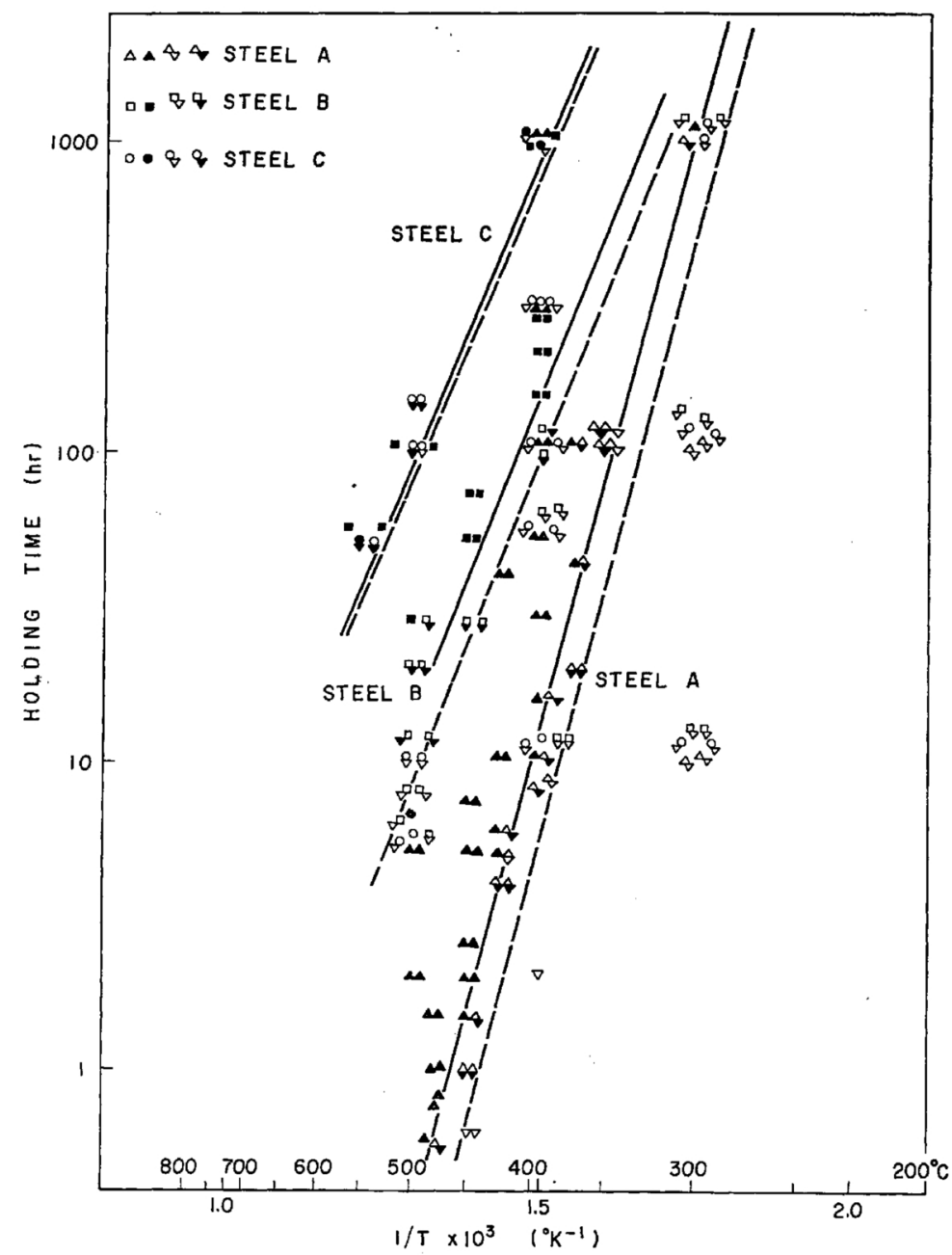

Fig. 6 Relation of embrittlement and heating temperature of three high strength steels after heating in $200 \mathrm{~kg} / \mathrm{cm}^{2}$ hydrogen.

$$
\begin{aligned}
& \text { - embrittlement limits by the reduction of area } \\
& \text { embrittlement limits by the impact value } \\
& \triangle \square O \cdots \text { non-embrittlement } \\
& \Delta \text { - ... embrittlement } \\
& \Delta \quad \cdots \text { non-embrittlement by the impact value (shows only a non- } \\
& \text { - ... embrittlement }\}_{\text {embrittlement zone by tensile tests) }}
\end{aligned}
$$

the impact test is somewhat shorter than that in the tensile test, presumably due to the difference in susceptibility to the micro-cracks produced within the structure.

A similar embrittlement process was observed for Steels B and C. The relationship between heating temperature and embrittlement beginning time of each steel is obtained. The time required for embrittlement beginning $(t)$ is obtained from the decreases in elongation, reduction of area, and impact values. The embrittlement limits are shown in Fig. 6 ; the ordinate is the holding time on a logarithmic scale, and the abscissa is the reciprocal of absolute temperature. In this case, the reduction in ductility more than $90 \%$ is regarded as embrittlement. From this result it is made clear that the embrittlement limit of each steel shows a linear relationship. Among the three steels, Steel A

\section{Discussion}

All the steels tested show embrittlement by heating in high pressure hydrogen at high temperatures with a tendency that the embrittlement beginning time becomes shorter with rising temperature. Such a tendency is most remarkable in Steel A, but Steel C is low in brittleness. As reported by Naumann(2), this seems due to the addition of carbide-forming element Mo in Steel C. It is also found that even in the same Mn-Si type steels as Steels A and B the effect of heat treatments upon hydrogen attack is considerable. According to Naumann's opinion, hydrogen attack is based on the permeability of hydrogen into the steel, resulting in the formation of methane by the reaction of hydrogen with the iron carbide. This process leads ultimately to 
cracking at grain boundaries. Therefore it is considered that the addition of carbide-stabilizing element Mo is effective to prevent the steel from hydrogen attack. Moreover, it appears that the steel whose structure was stabilized by heat treatment has a higher resistance to embrittlement than the as-rolled steel owing to the stabilizing of carbide.

As to the mechanism of the hydrogen attack, the above explanation is consistent with the present experimental results, but the details of this mechanism are not clear.

As the result of the present experiment, it is found that at a constant temperature and pressure there is an incubation time $(t)$ prior to embrittlement and after the embrittlement the steel loses the ductility in a short time by inner cracking accompanied by decarburization, and that a plot of the In $t$ vs. reciprocal of absolute temperature holds a linear relationship as shown in Fig. 6. Therefore, it may be considered that this reaction process has an activation energy. The values of the activation energy calculated by this linear relationship are about $36,000 \mathrm{cal} / \mathrm{mol}$ for Steel A and about $24,000 \mathrm{cal} /$ mol for Steels B and C; these values are far greater than that of hydrogen or carbon diffusion in the steels. Therefore, the predominant process is not clear. It is necessary to make a more detailed experiment to explain the mechanism of the embrittlement process. According to Weiner's report ${ }^{(7)}$ on the activation energy of the hydrogen attack, the value of $\mathrm{Al}$ killed steel in about $50 \mathrm{~kg} / \mathrm{cm}^{2}$ hydrogen is $14,600 \mathrm{cal} / \mathrm{mol}$, and is not equal to that of the present work.

\section{Conclusion}

(7) L. C. Weiner : Acta Met., 8(1960), 52.
Three high strength steels were heated in $200 \mathrm{~kg}$ / $\mathrm{cm}^{2}$ hydrogen at high temperatures, and the changes in their mechanical properties and structures were studied at room temperature. The results obtained are as follows :

(1) The steels which were heated in high pressure hydrogen at high temperatures became brittle and showed a remarkable decrease in the mechanical strength and ductility with the concurrent decrease in the hardness and carbon content, and many inner cracks occurred at grain boundaries. The fracture occurred from grain boundaries, and the fracture surfaces showed a grey intercrystalline fracture appearance.

(2) At a constant temperature and pressure, there is an incubation time $(t)$ prior to embrittlement, and after the embrittlement the ductility decrease remarkably in a short time. A plot of the $\ln t$ vs. reciprocal of absolute temperature holds a linear relationship.

(3) The activation energy of embrittlement by hydrogen attack was calculated, but the mechanism of the embrittlement remains unexplained.

(4) The addition of Mo, a carbide forming element, and the heat treatment such as normalizing and tempering increase the resistance to hydrogen attack and raise embrittlement limits.

\section{Acknowledgement}

The authors wish to express their sincere thanks to Dr. Kenji Ono, General Manager of the Hitachi Research Laboratory for his encouragement throughout this investigation and also wish to thank Mr. Iwao Takase for his help in the experimental work. 